\title{
A Monte Carlo Approach to the Approximation of Invariant Measures
}

F. Y. Hunt

U.S. DEPARTMENT OF COMMERCE

Technology Administration

National Institute of Standards

and Technology

Applied and Computational Mathematics Division Computing and Applied Mathematics Laboratory Gaithersburg, MD 20899

$Q C$ 



\section{A Monte Carlo Approach to the Approximation of Invariant Measures}

\section{F. Y. Hunt}

U.S. DEPARTMENT OF COMMERCE Technology Administration National Institute of Standards and Technology Applied and Computational Mathematics Division Computing and Applied Mathematics Laboratory Gaithersburg, MD 20899

January 1993

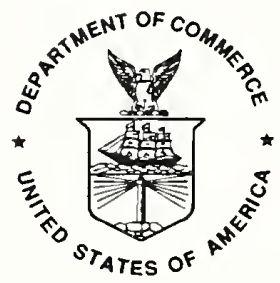

U.S. DEPARTMENT OF COMMERCE Ronald H. Brown, Secretary

NATIONAL INSTITUTE OF STANDARDS AND TECHNOLOGY

John W. Lyons, Director 



\title{
A MONTE CARLO APPROACH TO THE APPROXIMATION OF INVARIANT MEASURES
}

\author{
F. Y. Hunt \\ Computing and Applied Mathematics Laboratory \\ National Institute of Standards and Technology \\ Gaithersburg, MD 20899
}

January 28, 1993

\begin{abstract}
Approximation of absolutely continuous measures of maps of the interval and the closely related tasks of computing Lyapunov exponents and metric entropy are accomplished in principle by iterating the map to produce a sufficiently long trajectory. There is an alternative approach based on approximating the fixed point of the FrobeniusPerron operator. We present a Monte-Carlo implementation of the original piecewise constant method proposed by Ulam. This method has the advantage of not requiring explicit evaluation of the elements of the approximate Frobenius-Perron operator. Convergence rates of Ulam's method and some recently proposed higher order variants are discussed. Using the classical Bohman-Korovkin theorems of approximation theory the optimality of the rates are shown to be a consequence of the saturation phenomenon. Finally Ulam's scheme is used to estimate the leading Lyapunov exponent of a one dimensional map with an absolutely continuous measure. We propose an analytical criterion for comparing the results of using this method with an estimate obtained by iterating a long trajectory and illustrate its use in a numerical example.
\end{abstract}


1. Introduction. Given a non-singular mapping $\tau$ of a compact subset $X \subset \boldsymbol{R}$, it is of interest to determine whether or not an absolutely continous invariant measure exists, for such a measure is one indication of the chaotic behaviour of trajectories of the map. The existence of such a measure $\mu$ is equivalent to the existence of a fixed point $f^{*}$ of the Frobenius- Perron operator $P_{\tau}: L^{1}(X) \rightarrow L^{1}(X)$ and if

$$
P_{\tau} f^{*}=f^{*},
$$

$f^{*}$ (suitably normalized) is the density of $\mu$ with respect to Lebesque measure (m) and is called a stationary density. Since the pioneering work of Lasota and Yorke in [18] (1) has become an important strategy for establishing the existence of smooth invariant measures. Computing $f^{*}$ is also an important alternative to iterating $\tau$ and creating a histogram. Examples in [13] show that iteration produces roundoff and discretization effects that can lead to misleading results. Boyarsky and his coworkers have recently made progress in approximating measures associated with a class of expanding maps of the unit interval by using invariant measures of a Markov map that approximates the original map [8], [9]. These maps include the ones originally considered in [18]. The solution of (1) for Markov maps reduces to solving a matrix vector problem and the corresponding stationary densities were shown to converge to the stationary density of the original map by Gora and Boyarsky [8].

In 1960, Ulam [22] proposed approximating $P_{\tau}$ itself by a finite dimensional Markov operator, $P_{n}(\tau)$. In this setting $X=[0,1]$. He conjectured that the corresponding fixed point $f_{n}$ converges to $f^{*}$, provided $f^{*}$ exists. T.Y. Li proved this for the maps $\tau$ for which $\inf _{[0,1]}|\tau \prime|>2$. As in the previous approximation method finding $f_{n}$ can be accomplished by solving a matrix vector equation. However Ulam's method uses a simple uniform partition of the interval and for that reason, it has a natural generalization to domains in higher dimensions. Convergence in this setting was proved by Keller [14] for expanding maps. Chiu et. al. in [3], using a spectral approach obtained upper bounds on the rate of convergence with explicit constants. Earlier Hunt and Miller used spectral analysis and the Cauchy integral representation of the resolvent to show similar bounds on the rate of convergence when the operator $P_{\tau}$ is quasi-compact in $L^{1}$ [12]. Unfortunately this condition is not satisfied by many of the maps of the Lasota-Yorke type [5]. However under certain boundedness conditions randomly perturbed maps in $R^{n}$ are quasi-compact in $L^{1}(X)$ [11]. In this paper we will mainly be concerned with rates of convergence of Ulam's method and some recently proposed higher order variants 
given conditions on the smoothness of $f^{*}$. These rates provide error estimates for the method and help to answer the question: given a reasonable approximation of the map $\tau$ and its derivative can one obtain a good approximation of the Lyapunov exponent? A positive Lyanpunov exponent is one of the few unambiguous indications of chaos, and since the dynamics of many maps and differential equations in $\boldsymbol{R}^{\mathbf{n}}$ can be reduced to the dynamics of a one dimensional map, this question is important.

In $\S 2$ we describe Ulam's method and its implementation using a Monte Carlo calculation of $P_{n}(\tau)$.Using this approach one can avoid the problem of analytical evaluation which in general is quite difficult. In $\S 3$ convergence rates are discussed. Such rates were obtained by Chiu et. al. in the case when $f^{*}$ is of bounded variation or is in a Sobolev subspace of $\boldsymbol{C}^{1}$. We will obtain similar bounds using the classical BohmanKorovkin theorems of approximation theory. We propose that the optimality in the rate of convergence discussed by Chiu et. al. for the piecewise linear version of Ulam's method can be seen to be a consequence of a so-called saturation theorem (see [4]). This is also true of the piecewise quadratic version as well. These theorems imply a limitation in the speed of convergence independent of any extra smoothness $f^{*}$ might have, and this is evident when examining the numerical results in a recent paperof Ding and $\mathrm{Li}$ [7] on Markov finite approximations of the Frobenius-Perron operator. The errors using the quadratic version of the method are the same order of magnitude as the those of the linear method. We demonstrate in two numerical examples in $\S 4$ that the bounds on the rate of convergence obtained in $\S 3$ can be achieved in our Monte Carlo implementation although the error will be larger than methods based on exact analytical evaluations. In $\S 5$ we apply Ulam's method to the problem of computing the leading Lyapunov exponent of a one dimensional map. Bounds on the convergence rate of the method based on iteration can be obtained from extensions of Koksma's inequalitya [16]. These bounds do not take into account the effects of roundoff so, they apply under "ideal" conditions and for a large enough number of iterations. Based on these considerations a criterion for comparing Ulam's method and the iteration method under ideal conditions is presented and illustrated in a numerical example. Since the bounds are asymptotic in the number of iterations the comparison is a rough one. Nevertheless, the example shows that good results can be obtained with a modest amount of computation and more importantly the criterion gives a precise way of assessing the performance of Ulam's method or any other method to results obtained by iteration. 
This aproach applies not only to the Lyapunov exponent but also to any functional that is an expected value with respect to the invariant measure.

2. Numerical Implementation of Ulam's Method. Ulam's method is based on the approximation of $P_{\tau}$ by a Markov operator of finite rank. First introduce the finite dimensional subspace

$$
\triangle_{\mathbf{n}}=\left\{f \in L^{1}(X): f=\sum_{i=1}^{n} f_{i} \chi_{i}\right\}
$$

where $\chi_{i}$ is the indicator function of the ith subdivision $I_{i}$ in a partition of $X$, and $f_{i}$ are constants. We assume the measure of the subdivisions are all the same so that $m\left(I_{i}\right)=1 / n=h$. Let $\pi_{\mathbf{n}}$ be the operator on $L^{1}=L^{1}(X)$,

$$
\pi_{\mathbf{n}}: L^{1} \rightarrow \triangle_{\mathbf{n}}
$$

where $\pi_{\mathbf{n}} f=\sum_{i=1}^{n} f_{i} \chi_{i}$ and $f_{i}=1 / h \int_{I_{i}} f$. Let $P_{n}(\tau)=\pi_{\mathbf{n}} P_{\tau}$. If $\mathcal{D}=\left\{f \in L^{1}(X)\right.$ : $\left.f \geq 0, \int_{X} f=1\right\}$ is the set of densities then, $P_{n}(\tau)$, inheriting the properties of $P_{\tau}$, maps densities to densities and is therefore a Markov operator. Furthermore, $P_{n}(\tau) f \in \triangle_{\mathbf{n}}$ if $f \in \triangle_{\mathbf{n}}$, so the search for fixed points of $P_{n}(\tau)$ reduces to the solution of a matrix vector equation. Li proved the existence of a fixed point $f_{n}$ and proved the convergence of $\left\{f_{n}\right\}$ to $f^{*}$ when $\tau$ is an expanding piecewise $C^{2}$ map of the type considered by Lasota and Yorke. The defining relation for the operator $P_{\tau}$ is

$$
\int_{\tau^{-1}(A)} f=\int_{A} P_{\tau} f
$$

It is not hard then to show that the matrix representation of $P_{n}(\tau)$ is,

$$
\left[\widetilde{P_{n}(\tau)}\right]_{i j}=\frac{m\left(\tau^{-1}\left(I_{j}\right) \cap I_{i}\right)}{m\left(I_{i}\right)}
$$

Our implementation makes use of a Monte Carlo approximation of these elements as analytic evaluation may not be feasible. This is typically the case when $\tau$ has a complicated formula or when an analytic expression for $\tau$ is unavailable because for example it was obtained numerically or from an experiment. In each subinterval $I_{i}, \mathrm{M}$ points are selected at random from a uniform distribution. Call them $\left\{\boldsymbol{P}_{k}^{i}\right\}_{k=1,2, \cdots M}$. Then $\left\{\tau\left(\boldsymbol{P}_{k}^{i}\right)\right\}$ is calculated. Let $c_{i j}$ be the number of points $\tau\left(\boldsymbol{P}_{k}^{i}\right)$ in $I_{j}$. We then will have

$$
\frac{c_{i j}}{M} \approx \frac{m\left(\tau^{-1}\left(I_{j}\right) \cap I_{i}\right)}{m\left(I_{i}\right)}
$$


Let the resulting approximate matrix be called $\widehat{P_{n}(\tau)}$. The fixed points of $\widehat{P_{n}(\tau)}$ can be found by the usual matrix-vector methods. Now it was shown in [12] that if $\tau$ is ergodic the matrix $\widetilde{P_{n}(\tau)}$ is an irreducible,stochastic matrix. It follows that there is always a unique fixed point so for large $\mathrm{M}$ we expect the same conclusion holds for $\widehat{P_{n}(\tau)}$. The algorithm is highly parrallelizable, and for large $\mathrm{n}$, takes no more than a couple of minutes on a workstation.

3. Convergence. In this discussion we assume that $\mathrm{X}$ is the closed interval $[0,1]$, and that $P_{\tau}$ has 1 as a simple eigenvalue. Using the Cauchy integral representation of the projection onto the eigenspace for $\{1\}$, Chiu, et. al. proved the following upper bound on $\left\|f^{*}-f_{n}\right\|$, the $L^{1}$ norm of $f^{*}-f_{n}$.

Theorem 3.1 (Chiu,Du ANd Li [3]). Let $\tau$ satisfy in $f_{[0,1]}\left|\tau^{\prime}\right|>2$. Then $\left\|f^{*}-f_{n}\right\| \leq c \cdot\left\|f^{*}-\boldsymbol{Q}_{n} f^{*}\right\|$ where $c$ is a constant independent of $f_{n}$ and $f^{*}$.

c depends on the upper bound of the norms of the resolvent operators of $P_{\tau}$ and $P_{n}(\tau)$ and $\boldsymbol{Q}_{n}$ is the operator $\boldsymbol{\pi}_{\mathrm{n}}$, for the piecewise approximation originally considered by Ulam. Methods based on higher order approximations were developed by Ding and Li [7] and in these cases $\boldsymbol{Q}_{n}$ is an operator mapping $L^{1}(X)$ onto the finite- dimensional subspaces of piecewise linear and piecewise quadratic functions corresponding to the piecewise linear and piecewise quadratic approximations of $f^{*}$ considered by them. As will be shown below, Theorem 3.1 thus reduces the problem of estimating the error of Ulam's method and these related methods to a problem in classical approximation theory. That is, assuming $f^{*}$ has some degree of smoothness, upper bounds on the right hand side in theorem 3.1 can be obtained. Chiu,et.al. assumed $f^{*}$ was in a Sobolev subspace of $\boldsymbol{C}(0,1)$. Here Bohman-Korovkin theorems will be used to extend some of their results.

To apply the Bohman-Korovkin theorems in [4] we introduce the positive operator $\boldsymbol{U}_{n}: L^{1}(X) \rightarrow \triangle_{n} \quad \boldsymbol{U}_{n}(f, \cdot)=\sum_{k=1}^{n} \lambda_{k}(f) \chi_{k}(\cdot)$. For Ulam's method, $\lambda_{k}(f)$ is $f_{k}$ defined in equation (3). If we define the functions $e_{i}(x) \equiv x^{i}, i=0,1,2$, then one observes that,

$\boldsymbol{U}_{n}\left(e_{0}, \cdot\right)=e_{0}$

and for fixed $\mathrm{x}$, 


$$
\boldsymbol{U}_{n}(f(x), \cdot)=f(x) \boldsymbol{U}_{n}\left(e_{0}, \cdot\right)=f(x)
$$

An upper bound follows from the following Bohman-Korovkin inequality. In its proof we assume the continuity of $\mathrm{f}$, but we do not need the continuity of $\boldsymbol{U}_{n}(f, \cdot)$.

Proposition 3.2. If $f \in \boldsymbol{C}(X)$ then,

$$
\begin{array}{r}
\left|\boldsymbol{U}_{n}(f, x)-f(x)\right| \leq|f(x)|\left|e_{0}(x)-\boldsymbol{U}_{n}\left(e_{0}, x\right)\right| \\
+\left(\boldsymbol{U}_{n}\left(e_{0}, x\right)+\boldsymbol{U}_{n}\left(e_{0}, x\right)^{1 / 2}\right) \omega\left(f, \alpha_{n}(x)\right)
\end{array}
$$

$\alpha_{n}(x)=\boldsymbol{U}_{n}\left((t-x)^{2}, x\right)$ and $\omega(f, \cdot)$ is the modulus of continuity of $\mathrm{f}$.

The proof of the proposition closely follows along the lines of the proof of Shisha and Mond in [4] and is given to make our presentation self contained. We begin first with a preliminary lemma.

LemMa 3.3. Let,

$$
\boldsymbol{Q}_{n}(f, x)=\sum_{k=1}^{n} \lambda_{k}(f) h_{k}(x)
$$

where

$$
\lambda_{k}(f)=\sum_{i=1}^{p_{k}} \frac{b_{k i}}{h} \int_{I_{k i}} f
$$

with $\sum_{i=1}^{p_{k}} b_{k i}=1, h_{k}(x) \geq 0$ for all $x$, and $I_{k i}$ are partititon intervals. Then for each $x$,

$$
\boldsymbol{Q}_{\boldsymbol{n}}(|f|, x) \leq \boldsymbol{Q}_{\boldsymbol{n}}\left(|f|^{2}, x\right)^{\frac{1}{2}} \boldsymbol{Q}_{\boldsymbol{n}}\left(e_{0}, x\right)^{\frac{1}{2}}
$$

Proof: Apply the Cauchy-Schwarz inequality to the sum (11), the sum in equation (12) and the integral in (12)

Proof of Proposition 3.2 We observe that,

$$
\begin{array}{r}
|f(t)-f(x)| \leq \omega(f,|t-x|)=\omega\left(f, \delta \cdot|t-x| \delta^{-1}\right) \\
\leq \omega(f, \delta)\left(1+\delta^{-1}|t-x|\right)
\end{array}
$$

Now $\left|\boldsymbol{U}_{n}(f, x)-f(x)\right|=\left|\sum_{k=1}^{n}\left(\lambda_{k}(f)-f(x)\right) \chi_{k}(x)+\sum_{k=1}^{n} f(x) \chi_{k}(x)-f(x)\right|$.

Hence,using equation(8),

$$
\left|\boldsymbol{U}_{n}(f, x)-f(x)\right| \leq \boldsymbol{U}_{n}(|f(t)-f(x)|, x)+\left|\boldsymbol{U}_{n}\left(e_{0}, x\right)-e_{0}(x)\right||f(x)|
$$


Let $\delta>0$ be arbitrary. On using(15) the right hand side of (16) is

$$
\leq \omega(f, \delta)\left(\boldsymbol{U}_{n}\left(e_{0}, x\right)+\delta^{-1} \boldsymbol{U}_{n}(|t-x|, x)\right)+|f(x)|\left|\boldsymbol{U}_{n}\left(e_{0}, x\right)-e_{0}(x)\right|
$$

From lemma 3.3,

$$
\boldsymbol{U}_{n}(|t-x|, x) \leq\left(\boldsymbol{U}_{n}\left((t-x)^{2}, x\right)^{\frac{1}{2}}\left(\boldsymbol{U}_{n}\left(e_{0}, x\right)\right)^{\frac{1}{2}}\right.
$$

The right hand side is,

$$
\boldsymbol{U}_{n}\left(e_{0}, x\right)^{\frac{1}{2}} \alpha_{n}(x)
$$

and thus, on setting $\delta=\alpha_{n}(x)$,

$(20)\left|\boldsymbol{U}_{n}(f, x)-f(x)\right| \leq|f(x)|\left|\boldsymbol{U}_{n}\left(e_{0}, x\right)-e_{0}(x)\right|+\left(\boldsymbol{U}_{n}\left(e_{0}, x\right)+\left(\boldsymbol{U}_{n}\left(e_{0}, x\right)\right)^{\frac{1}{2}}\right) \omega(f, \delta)$

Since $\delta$ was arbitrary the conclusion follows from taking $\delta=\alpha_{n}(x)$ Q.E.D.

Using theorem 3.1 and equations (8), and (7) we have the following error estimate for the piecewise-constant version of Ulam's method:

Proposition 3.4 .

$$
\left\|f^{*}-f_{n}\right\| \leq c_{1}\left\|\omega\left(f, \alpha_{n}(x)\right)\right\|
$$

the constant $c_{1}$ is $2 c$.

We conjecture that Theorem 3.1 holds for a wider class of maps and for these, $f^{*}$ may be merely continuous. In these cases, Proposition 3.4 is useful. When $\mathrm{f}$ is Lipschitz, Proposition 3.4 implies that the error of the method is $\mathrm{O}(\mathrm{h})$. It is not hard to show that this is also the case when $\mathrm{f}$ is of bounded variation

Lemma 3.5. Suppose $f$ is defined on $[0,1]$ and is of bounded variation and let

$$
\boldsymbol{U}_{n} f=\sum_{i=1}^{n} f_{i} \chi_{i}
$$

where

$$
f_{i}=1 / h \int_{I_{i}} f
$$

and $\chi_{i}$ is the indicator function of the set $I_{i}$. Then,

$$
\left\|\boldsymbol{U}_{n} f-f\right\| \leq h \cdot \operatorname{Var}(f)
$$


where $\operatorname{Var}(f)$ is the variation of $f$ over $[0,1]$

Proof:

$$
\begin{array}{r}
\int_{0}^{1}\left|\boldsymbol{U}_{n} f-f\right| \leq \sum_{k=1}^{n} \int_{I_{k}}\left|f_{k}-f(x)\right| d x \\
\leq \sum_{k=1}^{n} \int_{I_{k}}|f(u(x))-f(x)| d x
\end{array}
$$

for some point $\mathrm{u}(\mathrm{x})$ in $I_{k}$ Now since the $I_{k}$ are disjoint except at the endpoints,

$$
\leq \sum_{k=1}^{n} \int_{I_{k}} \operatorname{Var}_{I_{k}}(f) d x=h \sum_{k=1}^{n} \operatorname{Var}_{I_{k}}(f)=h \operatorname{Var}(f)
$$

Q.E.D.

We will now examine some higher order methods introduced by Ding and Li. Let $\triangle_{n}^{1}=\left\{f \in L^{1}(X): f(x)=\sum_{i=1}^{n} f_{i} \phi_{i}(x)\right\}$ where $\left\{\phi_{i}\right\}_{i=0}^{n}$ are continuous piecewise linear functions with respect to the partition $\left\{I_{i}\right\}, i=1, \ldots, n$. Specifically,

$$
\phi_{i}(x)= \begin{cases}1+\frac{x-x_{i}}{h} & \text { if } x_{\imath-1} \leq x<x_{i} \\ 1-\frac{x-x_{i}}{h} & \text { if } x_{\imath} \leq x \leq x_{i+1} \\ 0 & \text { otherwise }\end{cases}
$$

where $\left[x_{i-1}, x_{i}\right]=[(i-1) h, i h]$ is $I_{i}$.

The operator $\boldsymbol{L}_{n}: L^{1}(X) \rightarrow \triangle_{n}^{1}$ is defined by; $\boldsymbol{L}_{n}(f, x)=\sum_{i=0}^{n} f_{i} \phi_{i}(x)$ where

$$
f_{i}=\frac{1}{2 h}\left(\int_{I_{i}} f+\int_{I_{i+1}} f\right) \quad 1 \leq i \leq n-1
$$

and

$$
f_{0}=1 / h \int_{I_{\mathrm{i}}} f \quad f_{n}=1 / h \int_{I_{n}} f
$$

In analogy with Ulam's piecewise linear method, let $\widetilde{\boldsymbol{\pi}_{n}} f(x)=\boldsymbol{L}_{n}(f, x)$, so that the finite Markov approximation to $P_{\tau}$ becomes $P_{n}(\tau)=\widetilde{\pi}_{n} P_{\tau}$. Ding and Li showed that $P_{n}(\tau): \triangle_{n}^{1} \rightarrow \triangle_{n}^{1}$, has a fixed point $f_{n}^{L} \in \triangle_{n}^{1} \cap \mathcal{D}$, and the the sequence $\left\{f_{n}^{L}\right\}$ converges. They assumed firstly that $\tau$ was piecewise $C^{2}$, map with $\inf _{[0,1]}\left|\tau^{\prime}\right|>2$, and secondly that there is a unique stationary density. Chiu, Du and Li obtained error bounds for $f^{*} \in \boldsymbol{W}^{\boldsymbol{2}, 1}(0,1) \cap \boldsymbol{C}^{1}(0,1)$, where $\boldsymbol{W}^{2,1}(0,1)$ is the space of absolutely integrable 
functions with absolutely integrable first and second derivatives. Given the conclusions of Theorem 3.1, they show that there is a constant c such that

$$
\left\|f^{*}-f_{n}^{L}\right\| \leq \frac{c}{n^{2}}=c h^{2}
$$

$$
\text { for } \mathrm{n} \text { large enough }
$$

The Bohman-Korovkin inequality we will use is

Lemma 3.6. Let $f \in C^{1}(X)$. Then,

$$
\begin{array}{r}
\left|\boldsymbol{L}_{n}(f, x)-f(x)\right| \leq|f(x)|\left|\boldsymbol{L}_{n}\left(e_{0}, x\right)-e_{0}(x)\right|+\left|f^{\prime}(x)\right|\left|\boldsymbol{L}_{n}(t-x, x)\right|+ \\
+\left\{\boldsymbol{L}_{n}\left(e_{0}, x\right)^{1 / 2}+1\right\} \alpha_{n}(x) \omega\left(f^{\prime}, \alpha_{n}(x)\right)
\end{array}
$$

where $\alpha_{n}(x)=\boldsymbol{L}_{n}\left(|t-x|^{2}, x\right)^{1 / 2}$

The proof is very similar to that of Proposition 3.2 A direct calculation shows that $\alpha_{n}(x) \leq$ const $\cdot \frac{1}{n}$. It is also not hard to see that $\boldsymbol{L}_{n}\left(e_{0}, x\right)=e_{0}(x)$, and $\boldsymbol{L}_{n}(t-x, x)=$ 0 .We then will have the following estimate for the piecewise linear method.

Proposition 3.7. Let the conclusions of Theorem 3.1 be satisfied. Further suppose that $f^{*}$ is in $C^{1}(X)$. Then,

$$
\left\|f^{*}-f_{n}^{L}\right\| \leq \mathrm{const} \cdot h\left|\omega\left(f^{* \prime}, h\right)\right|
$$

One can derive other conditions for the $\mathrm{O}\left(h^{2}\right)$ rate of convergence stated in equation (29), when $f^{* \prime}$ is continous.

Proposition 3.8. The following conditions imply that (29) holds.

1. $f^{* \prime}$ is Lipschitz

2. $f^{* \prime \prime}$ is $\boldsymbol{L}^{\infty}(0,1)$

Proposition 3.7 shows that as long as $f^{*}$ is $C^{1}$, we can expect an improvement in the error over the original piecewise constant Ulam method. This is quite evident in numerical computations of Ding and Li (see tables 1-4 in [7]). However the degree of improvement will ( not surprisingly) depend on the smoothness of $f^{*}$. For example if $f^{*}$ is of bounded variation, then Chiu et. al. found an upper bound that is $\mathrm{O}(\mathrm{h})$. This is however already achieved in the piecewise constant case so the implication is that there is no improvement. And if $f^{*}$ is merely Holder continuous, then Proposition 3.7 will lead to upper bounds that are slower than $\mathrm{O}\left(h^{2}\right)$. 
Chiu et. al. briefly note that the upper bound in (29) is "optimal" for the piecewise linear method; moreover the results of computations using a piece-wise quadratic approximation developed in reference [7] suggests that the rate of convergence is of the same order of magnitude as the piecewise linear method. A possible explanation for this lies in the following saturation theorem due to DeVore [4] that highlights the limitations of this approach to approximation of $f^{*}$.

Theorem 3.9 (DeVore [4]). Let $f^{*} \in \boldsymbol{C}(0,1)$, and let $\boldsymbol{Q}_{\boldsymbol{n}}, n=1,2, \cdots$ be a sequence of positive linear operators, $\boldsymbol{Q}_{\boldsymbol{n}}: \boldsymbol{C}(0,1) \rightarrow \boldsymbol{C}(0,1)$.

If $\mu_{n}(x)=1 / 2 Q_{n}\left((t-x)^{2}, x\right)>0$, and if for each $x$,

$$
e_{i}(x)-Q_{n}\left(e_{i}, x\right)=o_{x}\left(\mu_{n}(x)\right) \quad i=0,1
$$

then

$$
f(x)-Q_{n}(f, x)=o_{x}\left(\mu_{n}(x) \text { for } x \in[0,1]\right.
$$

iff $f$ is linear

$o_{x}$ means that the little o behaviour is allowed to be pointwise in $\mathrm{x}$. Now for the linear method where $Q_{n}=L_{n}, \mu_{n}(x)=$ const. $h^{2}>0$ by calculation. It is also true that $\boldsymbol{L}_{n}\left(e_{i}, x\right)=e_{i}(x), i=0,1$, so we conclude that

Proposition 3.10. Suppose $f^{*}$ is in $\boldsymbol{C}^{1}(0,1)$ but is not linear. Then,

$$
f^{*}(x)-\boldsymbol{L}_{n}\left(f^{*}, x\right) \neq o_{x}\left(h^{2}\right)
$$

This suggests that the accuracy of the piecewise linear method does not improve indefinitely with the smoothness of $f^{*}$

Let us turn now to the piecewise quadratic method introduced in [7]. Here we consider the finite dimensional space $\triangle_{n}^{2}=\left\{f \in L^{1}(0,1): \sum_{k=0}^{2 n} f_{k} \phi_{k}\right\}$. $\phi_{k}$ are continuous piecewise quadratic polynomials. They are defined as:

$$
\phi_{2 i}(x)= \begin{cases}\left(1+\frac{x-x_{i}}{h}\right)^{2} & \text { if } x_{i-1} \leq x<x_{i} \\ \left(\frac{x-x_{i}}{h}-1\right)^{2} & \text { if } x_{i} \leq x<x_{i+1} \\ 0 & \text { otherwise }\end{cases}
$$


when $\mathrm{k}=2 \mathrm{i}$.

When $k=2 \mathrm{i}-1$, the formula for $\phi_{k}$ is;

$$
\phi_{2 i-1}(x)= \begin{cases}2\left(\frac{x-x_{i}}{h}\right)\left(1-\frac{x-x_{i}}{h}\right) & \text { if } x_{i-1} \leq x<x_{i} \\ 0 & \text { otherwise }\end{cases}
$$

The operator mapping $L^{1}(0,1)$ to $\triangle_{n}^{2}$ is defined by $\boldsymbol{S}_{n}(f, x)=\sum_{k=0}^{2 n} f_{k} \phi_{k}$ where the constants $f_{k}$ are

$$
\begin{aligned}
& f_{2 i-1}=\frac{1}{h} \int_{I_{i}} f \quad i=1, \ldots n \\
& f_{2 i}=\frac{1}{2 h}\left(\int_{I_{i}} f+\int_{I_{i+1}} f\right) \quad i=1, \ldots, n \\
& f_{0}=\frac{1}{h} \int_{I_{1}} f \\
& f_{2 n}=\frac{1}{h} \int_{I_{n}} f
\end{aligned}
$$

We note that $\boldsymbol{S}_{n}: \boldsymbol{C}(0,1) \rightarrow \boldsymbol{C}(0,1)$.

As in the piecewise linear case Ding and Li showed that fixed point of the Markov finite approximating operator

$$
P_{n}(\tau) f=S_{n} P_{\tau} f
$$

converges to $f^{*}$ when $\tau$ satisfies the same conditions as in the piecewise linear case. One can show by calculation that $e_{i}(x)-\boldsymbol{S}_{n}\left(e_{i}, x\right)=0, i=0,1$, and $\mu_{n}(x)=O\left(h^{2}\right)$ as in Theorem 3.9.

Proposition 3.11. The conclusion of Proposition 3.10 also applies to the piecewise quadratic method defined in equation (39).

Whatever the degree of smoothness of $f^{*}$, the rate of convergence for the piecewise quadratic method is not faster than $O\left(h^{2}\right)$. This could explain the apparent similarity in rates of the piecewise linear and piecewise quadratic caculations in [7]. We note that $\boldsymbol{S}_{n}(f, x) \neq f(x)$ when $\mathrm{f}$ is a quadratic function with $f^{\prime \prime} \neq 0$. This suggests that saturation results from the fact that the integrals used in the approximating polynomials are 
of limited accuracy [21] and that using splines may give better results. (See [6] for convergence rates and numerical results for approximations based on Galerkin projections, where the same phenomenon seems to be present.)

4. Numerical Results. We turn now to a discussion of some numerical reults obtained from the Monte Carlo implementation of Ulam's method described in section $\S 1$. The theoretical analysis in the preceding sections neglected the error due to the use of Monte Carlo approximations of the elements of $P_{n}(\tau)$.There will be an additional error as can be seen in table 1 . The map $\tau_{1}(x)=1 / 2+\left(1 / 8-2\left|x-\frac{1}{2}\right|^{3}\right)^{1 / 3}$ is not of Lasota-Yorke type (it is conjugate to one) and doesn't satisy the hypothesis of Theorem 3.1. Nevertheless the stationary density can be calculated.The second column gives the errors for the Monte Carlo method and the third column lists the $L^{1}$ errors for the method based on analytical evaluation of the elements [7] of $P_{n}(\tau)$

TABLE 1

ERROR for $\tau_{\mathbf{1}}$

$\begin{array}{ccc}\mathrm{n} & \text { Monte Carlo } & \text { exact method } \\ 8 & .3648 & .22924 \\ 16 & .1989 & .10271 \\ 32 & .0979 & .05257 \\ 64 & .048 & .02564 \\ 128 & .0368 & .01337 \\ 256 & .0196 & .00645\end{array}$

The number of Monte Carlo test points used in the calculation was $M=1,000$ per box.

It is interesting to note that the Monte Carlo error is $\mathrm{O}(\mathrm{h})$ in this example, consistent with the theoretical bound in $\S 3$. We conjecture then, that these upper bounds hold for a wider class of maps. This rate is also achieved in a second example[10];

$$
\tau_{2}(x)= \begin{cases}1 / \sqrt{2}-\sqrt{2}\left|\frac{1}{2}-x\right| & \text { if } x \in[0,1 / \sqrt{8}] \cup[1-1 / \sqrt{8}, 1] \\ 1-1 / \sqrt{2} \sqrt{1-(1-|1-2 x|)^{2}} & \text { if } x \in[1 / \sqrt{8}, 1-1 / \sqrt{8}]\end{cases}
$$




\section{TABLE 2}

\begin{tabular}{cr}
\multicolumn{2}{c}{ ERROR for $\tau_{\mathbf{2}}$} \\
$\mathrm{n}$ & Monte Carlo \\
8 & .216 \\
16 & .1208 \\
32 & .0576 \\
64 & .0295 \\
128 & .0171
\end{tabular}

The number of Monte Carlo test points used in this second calculation was $M=8,000$ per box. Explicit formulae for the stationary densities are known in these cases. They are $f_{1}^{*}(x)=12\left(x-\frac{1}{2}\right)^{2}$ and $f_{2}^{*}(x)=2-|2-4 x|$ for $\tau_{1}$ and $\tau_{2}$ respectively. Graphical comparisons between the approximations and exact densities can be found in the appendix.

5. Approximation and Iteration: A Rough Comparison. We return to the question posed in the introduction concerning the accurate computation of the Lyapunov exponent of a one dimensional map. If several simplifying assumptions are made, one can make a comparison between the accuracy of computation by iterating as opposed to approximating the invariant measure and then integrating. First for simplicity, assume that $f^{*}=1$, and secondly that $\log \left(\left|\tau^{\prime}\right|\right)$ is of bounded variation. We neglect any possible error in its computation. Comparison of the methods will be made under the best of conditions in that we will also assume that there is no roundoff error incurred by iterating. Note however that if there is a positive Lyapunov exponent, roundoff error is likely because small perturbations will on average grow larger as iteration proceeds. The following definition will be used in this discussion.

Definition: Given a sequence $t_{1}, t_{2}, \ldots$ of numbers in $[0,1]$, the discrepancy of the first $\mathrm{N}$ terms of the sequence is,

$$
D_{N}=\sup _{[a, b) \subseteq[0,1]}|\sharp[a, b) / N-(b-a)|
$$

where $\sharp[a, b)$ is the number of $t_{i}$ 's lying in $[a, b)$. The concept of discrepancy plays an important role in quasi-Monte Carlo integration and the study of uniform distribution of sequences in $[0,1]$. J.P.Lambert noted the connection with iteration of ergodic 
maps.([17])

It is known that $\left\{t_{i}\right\}_{i=1}^{\infty}$ is uniformly distributed in $[0,1]$ iff $\lim _{N \rightarrow \infty} D_{N}=0$. Now let $\phi$ be a function of bounded variation. Given a uniform invariant measure the goal of both methods is to estimate $\int_{0}^{1} \phi(t) d t$. The upper bound on the error of finite iteration follows from

Lemma 5.1 (KoKsma's INequality). Let $t_{1}, t_{2}, \ldots t_{N}$ be a finite sequence with discrepancy $D_{N}$. Then,

$$
\left|\int_{0}^{1} \phi(t) d t-1 / N \sum_{i=1}^{N} \phi\left(t_{i}\right)\right|=e_{I}(N) \leq V(\phi) D_{N}
$$

Setting $\phi=\log \left|\tau^{\prime}\right|$,we see that the error in calculating the Lyapunov exponent from iteration is $O\left(D_{N}\right)$. The problem of lower bounds on $D_{N}$ has been studied extensively and the result of Schmidt (see [16]) is that there is a lower bound on the rate at which $D_{N}$ can go to zero.For any infinite sequence in $\boldsymbol{R}, N D_{N}>c \log (N)$ for infinitely many $\mathrm{N}$ with $c=(66 \log 4)^{-1}$. Thus the fastest possible rate at which $D_{N}$ can approach zero is $O(\log (N) / N)$. Choosing a sequence of grid sizes $h=1 / n$ for which $e_{U}=\left\|f^{*}-f_{n}\right\|$, the error in the Ulam approximation satisfies $e_{U} \leq c \log (N) / N$,one can call the Ulam approximation equivalent to iterating $\tau \mathrm{N}$ times under optimal conditions. Of course $e_{I}$ may be much smaller than the upper bound $\log (N) / N$ particularly if $\mathrm{N}$ is small, so the comparison is rough. It becomes precise if it can be determined that $D_{N}$ has order of magnitude $\log (N) / N$.

We close with some numerical illustrations. Consider a piecewise linear function of the unit interval $\tau_{3}$ as depicted in figure 1 .The function is a Markov map with respect to the partition $\mathcal{P}=\left\{\left[a_{0}, a_{1}\right),\left[a_{1}, a_{2}\right),\left[a_{2}, a_{3}\right),\left[a_{3}, a_{4}\right]\right\}$, where $a_{0}=0<a_{1}<$ $a_{2}<a_{3}<a_{4}=1$. Boyarsky and Scarowsky in [1] proved that piecewise monotone Markov maps of the intervai satisfying an expanding condition including $\inf f_{[0,1]}|\tau \prime|>1$, have unique absolutely continuous invariant measures. In the piecewise linear case the density of these measures are functions that are piecewise constant on the intervals of the partition. In [2] Boyarsky used this fact to develop a method for estimating the Lyapunov exponent of a piecewise expanding map. The leading Lyapunov exponent of $\tau_{3}$ can be calculated exactly so the accuracy of the Ulam approach can be tested in this case. Table 3 column 2 shows the error as a function of the number of grid boxes 
$n$. The intermediate endpoints were $a_{1}=1 / G^{3}, a_{2}=(1.9) * a_{1}, a_{3}=(3.25) * a_{1}$, where $G=\frac{1+\sqrt{(5)}}{2}$. With this choice, $\tau_{3}$ satisfies the conditions of Boyarsky and Scarowsky's theorem. The stationary density (normalized so that $\int_{0}^{1} f^{*} d m=1$ ) is,

$$
f_{3}^{*}(x)= \begin{cases}1.0530 \ldots & \text { if } 0 \leq x<a_{1} \\ 0.6762 \ldots & \text { if } a_{1} \leq x<a_{2} \\ 1.1269 \ldots & \text { if } a_{2} \leq x<a_{3} \\ 1.0679 \ldots & \text { if } a_{3} \leq x \leq 1\end{cases}
$$

Let $\left\{t_{1}, t_{2}, \ldots\right\}$ be a sequence of iterates of $\tau_{3}$. We assume that any initital transients have been discarded so that the $t_{i}$ are effectively distributed with density $f^{*}$. If the corresponding distribution function is $F^{*}$ recall that $F^{*}\left(t_{1}\right), F^{*}\left(t_{2}\right), \ldots$ is a sequence that has a uniform distribution. We can apply the results of our previous discussion to this sequence. Now,

$$
\int_{0}^{1} \phi(t) f^{*}(t) d t=\int_{0}^{1} \phi \circ F^{-1}(F(t)) d F(t)=\int_{0}^{1} \phi \circ F^{-1}(y) d y
$$

and also $\frac{1}{N} \sum_{0}^{N-1} \phi\left(t_{i}\right)=\frac{1}{N} \sum_{0}^{N-1} \phi \circ F^{-1}\left(y_{i}\right)$ where $y_{i}=F\left(t_{i}\right)$.

$\phi \circ F^{-1}$ is certainly of bounded variation if $\phi$ is and hence Koksma's inequality can be used to bound $\frac{1}{N} \sum_{0}^{N-1} \phi\left(t_{i}\right)-\int_{0}^{1} \phi(t) f^{*}(t) d t$. The right hand side will involve the variation of $\phi \circ F^{-1}$ and $D_{N}$ the discrepancy of the sequence $\left\{F\left(t_{i}\right)\right\}$.

TABLE 3

\section{COMPARISON OF LYAPUNOV EXPONENT COMPUTATION METHODS}

$\begin{array}{ccccc}\text { number of subdivisions } & \begin{array}{c}\text { Ulam error } \\ \text { (absolute value) }\end{array} & \begin{array}{c}\text { average iteration } \\ \text { error }\end{array} & \begin{array}{c}\text { number of } \\ \text { iterations } N\end{array} & \log (N) / N \\ 16 & .0019 & .008 & 100 & .046 \\ 32 & .0186 & .002 & 500 & .012 \\ 40 & .0053 & * & * & * \\ 64 & .0039 & .0016 & 2000 & .0038 \\ 128 & .0015 & * & * & *\end{array}$

In estimating the Lyapunov exponent by iterating the map, 20-25 initial values are used and column 3 of Table 3 shows the average error as a function of the number of iterations. The exact value is $\lambda=.4109964 \ldots$.. 
The $a_{i}^{\prime} s$ in $\tau_{3}$ were chosen to minimize the roundoff errors arising from a finite binary representation so the iteration errors are pretty good. After $N=2000$ iterations it is probably safe to say that $D_{N}$ has order of magnitiude $\log (N) / N$. Table 3 illustrates the point that the overall performance of Ulam's method for $f_{3}^{*}$ is comparable to iteration under ideal conditions and if we use the comparison criteria described earlier, $N=2000$ iterations is equivalent to using Ulam's method with $40 \leq n \leq 64$ subdivisions. If we used the bounds obtained in $\S 3$ on $e_{U}$ to bound the the Ulam error in Table 3 we would have obtained the more conservative $n=128$ subdivisions. The computations for column 2 were performed with approximations to the invariant density using the methods described in $\S 2$ with $M=2000$ Monte Carlo points. The reason for the small error at $n=16$ is not clear to us. The next example illustrates the chief advantage of computing Lyapunov exponents with invariant measures: accurate results can be obtained in cases where roundoff error is a significant problem. For many initial values, iterating a map $\tau_{4}$ with $a_{1}=1 / 4, a_{2}=1 / 2, a_{3}=3 / 4$, produces a sequence that is attracted to the periodic orbit determined by the $\left\{a_{i}\right\}$, thereby resulting in an extremely inaccurate estimate of the Lyapunov exponent. Given that $f^{*}$ is

$$
f_{4}^{*}(x)= \begin{cases}8 / 7 & \text { if } 0 \leq x<1 / 4 \\ 4 / 7 & \text { if } 1 / 4 \leq x<1 / 2 \\ 8 / 7 & \text { if } 1 / 2 \leq x<3 / 4 \\ 8 / 7 & \text { if } 3 / 4 \leq x \leq 1\end{cases}
$$

the exact value of the exponent is $\frac{4}{7} \log (2)=.396084 \cdots$, Table 4 gives the errors for Ulam's method, Table 5 displays the results of iterating 25 equally spaced points and averaging the results. Boyarsky's method, based on the construction of Markov partitions, yielded exact results for every partition used. Note that to obtain the value in [2], the exact value must be divided by $\log (2)$. Despite the superior performance of Boyarsky's method on Markov maps, the advantage of this implementation of Ulam's method is that uniform partitions are easier to calculate especially in higher dimensions. This will be explored in a forthcoming paper. 


\section{TABLE 4}

ULAM'S METHOD: ERRORS IN ESTIMATING $f_{4}^{*}$
$\begin{array}{cl}\text { number of subdivisions } & \text { Ulam error } \\ \mathrm{n} & \text { (absolute value) } \\ 16 & .0012 \\ 32 & 9.95 \mathrm{e}-04 \\ 64 & 3.12 \mathrm{e}-04\end{array}$

TABLE 5

ITERATION ERROR for $f_{4}^{*}$

number of iterations average iteration error

(25 points)

50

.023

100

.025

500

.100

1000

.114

\section{REFERENCES}

[1] A. Boyarsky, M. Scarowsky, On A Class of Transformations Which Have Absolutely Continuous Invariant Measures, Transactions of the American Mathematical Society, 255, pp.243262,1979

[2] A. Boyarsky, A Matrix Method for Estimating the Liapunov Exponent of One-Dimensional Systems, J. Stat. Phys., 50, No. 1/2, pp. 213-223, 1988

[3] C. ChIU, Q. DU, T. Y. LI, Error Estimates of the Markov Finite Approximation of the FrobeniusPerron Operator, preprint 1991, to appear J.Nonlinear Analysis

[4] R. A. DeVore, The Approximation of Continuous Functions by Positive Linear Operators, Lecture Notes in Mathematics No. 293, Springer Verlag, Berlin, Heidelberg, New York, 1972

[5] J. DING, Q. DU , T.Y. LI, The spectral analysis of the Frobenius-Perron operator, preprint 1992

[6] J. Ding, Q. Du, T. Y. LI, High Order Approximation of the Frobenius-Perron Operator to appear Appl. Math. Comp. 1993

[7] J. Ding, T. Y. LI, Markov Finite Approximation of Frobenius- Perron Operator, Nonlinear Analysis, Theory, Methods, and Applications, 17, No. 8, pp. 759-772, 1991

[8] P. Gora, A. Boyarsky, Compactness of Invariant Densities for Families of Expanding Piecewise Monotonic Transformations, preprint 1987 
[9] P. Gora,A. Boyarsky,H. Proppe, Constructive Approximations to Densities Invariant under Non-expanding Transformations,J.Stat.Phys., 51,pp. 179-194, 1987

[10] S. Grossmann, S. Thomae, Invariant Distributions and Stationary Correlation Functions of One-Dimensional Discrete Processes, Z.Naturforsch., 32a, pp. 1353-1363,1977

[11] F. Y. Hunt, Approzimating the Invariant Measures of Randomly Perturbed Dissipative Maps, preprint 1992

[12] F. Y. Hunt, W. M. Miller, On the Approximation of Invariant Measures, J.Stat.Phys.,66, No.1/2, pp. 535-548,1992

[13] R. V. Jensen, E. R. Jessup, Statistical Properties of the Circle Map, J.Stat.Phys., 43, No.1/2,pp. 369-389, 1986

[14] G. Keller, Stochastic Stability in Some Chaotic Dynamical Systems, Monat.Math., 94, pp. 313333

[15] U. Krengel, Ergodic Theorems, Walter de Gruyter, Berlin, New York, 1985

[16] L .Kuipers,H .Neiderreiter, Uniform Distribution of Sequences, John Wiley and Sons, New York, 1974

[17] J .P .LAMBerT, Quasi-Monte Carlo, low discrepancy sequences, and ergodic transformations, J.Comp.and Appl. Math.,12\&13,pp.419-423,1985

[18] A. Lasota, J. Yorke, On the Existence of Invariant Measures for Piecewise Monotonic Transformations, Trans.Am.Math.Soc.,186,pp. 481-489, 1973

[19] T. Y .LI, Finite approximation for the Frobenius-Perron operator.A solution to Ulam's conjecture,J.Approx.Theory,17,pp. 177-186, 1976

[20] M. RYchlik, Bounded Variation and Invariant Measures, Studia Math., 76, pp. 69-80, 1983

[21] H. Shapiro, Approximation Theory, Van Nostrand

[22] S.Ula , Problems in Modern Mathematics, Interscience Publishers, New York, 1960 












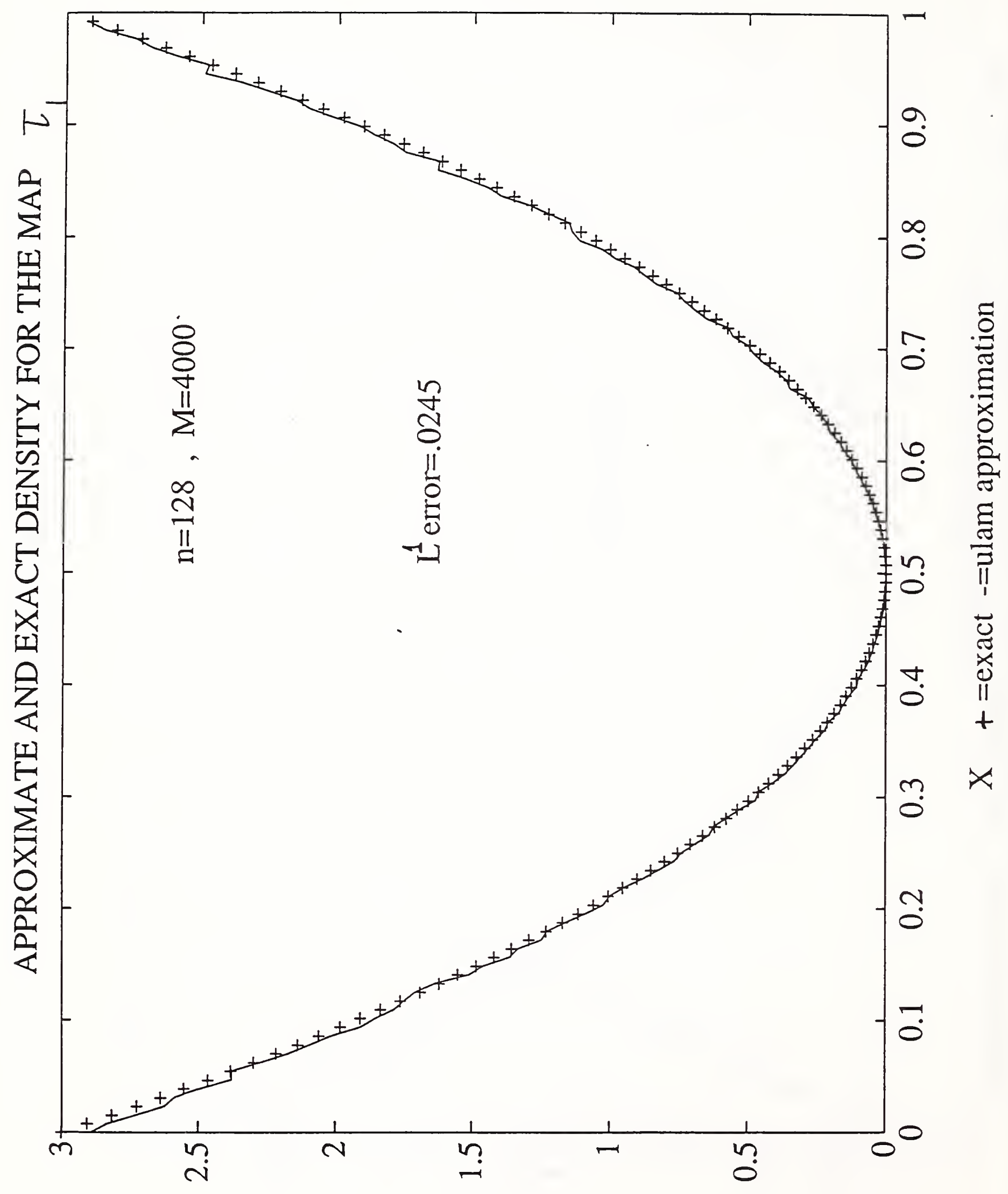




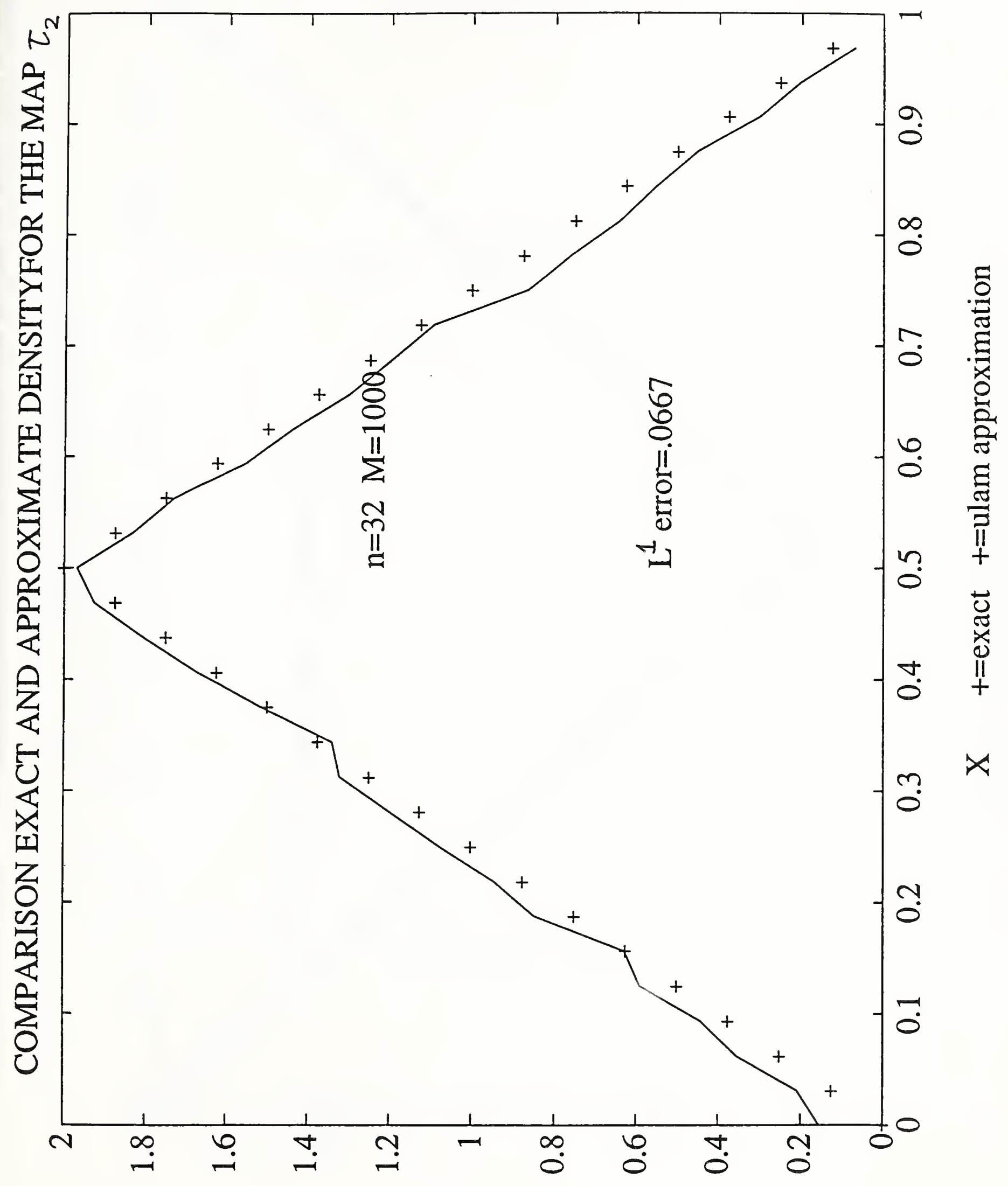




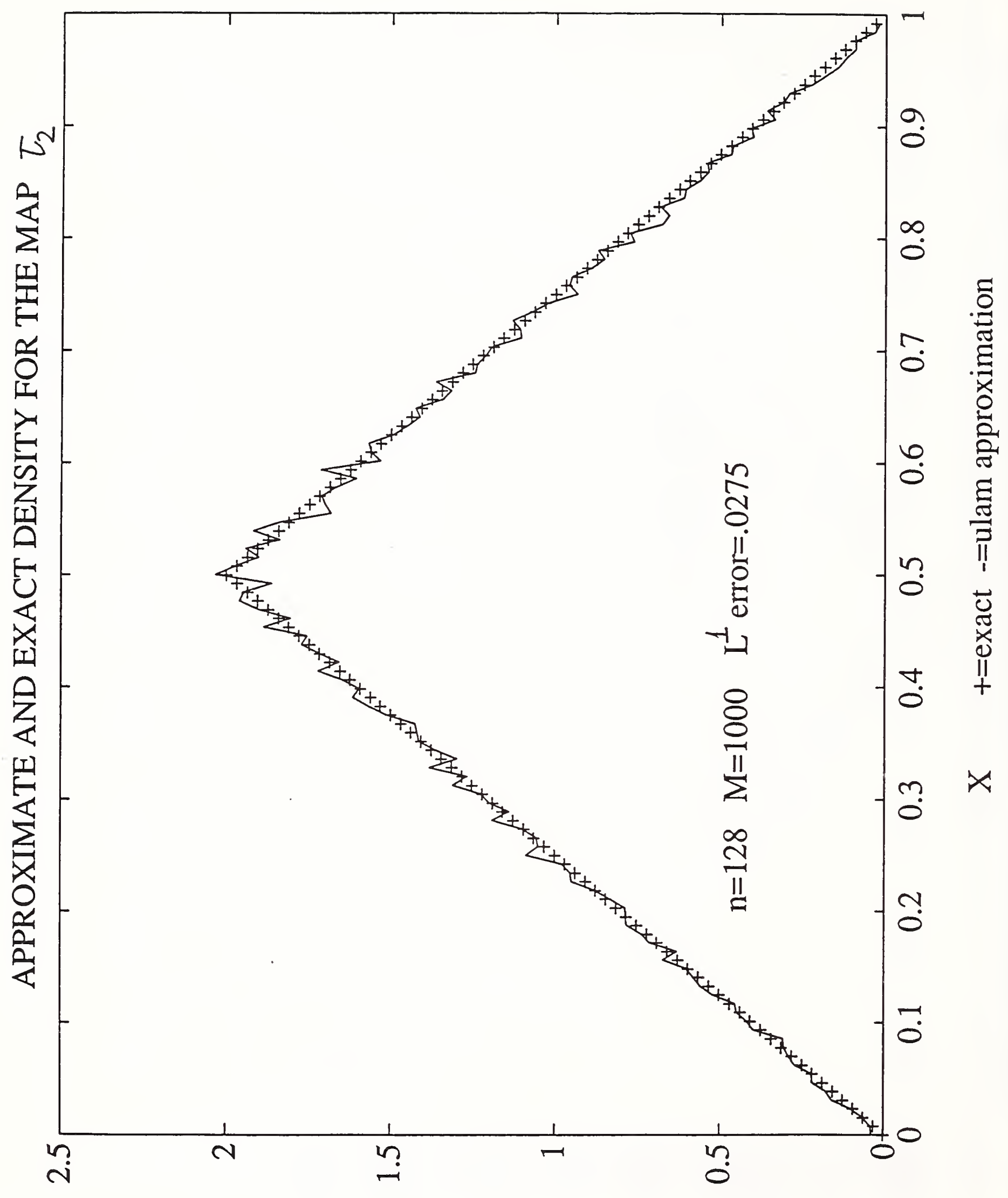


FIGURE 1





1 